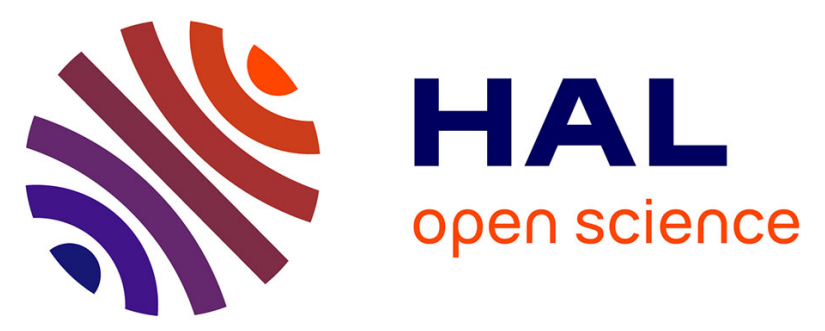

\title{
A closed-loop prescriptive maintenance approach for an usage dependent deteriorating item - Application to a critical vehicle component
}

Pedro Dias Longhitano, Khaoula Tidriri, Christophe Bérenguer, Benjamin Echard

\section{To cite this version:}

Pedro Dias Longhitano, Khaoula Tidriri, Christophe Bérenguer, Benjamin Echard. A closed-loop prescriptive maintenance approach for an usage dependent deteriorating item - Application to a critical vehicle component. ESREL 2021 - 31st European Safety and Reliability Conference, Sep 2021, Angers, France. pp.2465-2472, 10.3850/978-981-18-2016-8_545-cd . hal-03443125

\section{HAL Id: hal-03443125 \\ https://hal.science/hal-03443125}

Submitted on 23 Nov 2021

HAL is a multi-disciplinary open access archive for the deposit and dissemination of scientific research documents, whether they are published or not. The documents may come from teaching and research institutions in France or abroad, or from public or private research centers.
L'archive ouverte pluridisciplinaire HAL, est destinée au dépôt et à la diffusion de documents scientifiques de niveau recherche, publiés ou non, émanant des établissements d'enseignement et de recherche français ou étrangers, des laboratoires publics ou privés.

\section{(ㅇ)(1) $\$$}

Distributed under a Creative Commons Attribution - NonCommercial - NoDerivatives 44.0 


\title{
A closed-loop prescriptive maintenance approach for an usage dependent deteriorating item - Application to a critical vehicle component
}

\author{
Pedro D. Longhitano \\ Univ. Grenoble Alpes, CNRS, Grenoble INP, GIPSA-Lab, Grenoble, France \\ Volvo Group Trucks Technology, Saint-Priest, France E-mail: pedro.longhitano@volvo.com
}

\author{
Khaoula Tidriri \\ Univ. Grenoble Alpes, CNRS, Grenoble INP, GIPSA-Lab, Grenoble, France \\ E-mail: khaoula.tidriri@grenoble-inp.fr
}

\section{Christophe Bérenguer}

Univ. Grenoble Alpes, CNRS, Grenoble INP, GIPSA-Lab, Grenoble, France

E-mail: christophe.berenguer@grenoble-inp.fr

\author{
Benjamin Echard \\ Volvo Group Trucks Technology, Saint-Priest, France E-mail: benjamin.echard.2@volvo.com
}

Connectivity and the huge data availability today open doors for modern and adaptable maintenance solutions. Nowadays, clients have the desire, not only to avoid breakdowns, but also to find out how to use their systems in order to reduce the overall exploitation cost and expand the remaining useful life. Researchers of different areas have worked to achieve such goal, but a complete solution that quantitatively assesses for different actions, costs and the degradation evolution was not proposed. In this paper, we will consider the example of a fleet of vehicles. We will model different operational costs as well as the degradation trajectory of a real component, and account for two possible actions: mission planning and maintenance operations scheduling. We highlight how those two actions are connected and propose a method for finding the optimal usage of the fleet. Finally, through simulations, we compare it to a maintenance/exploitation policy that mimics real fleet usage.

Keywords: Maintenance, decision making, multi-objective optimization, degradation and reliability models, automotive sector.

\section{Introduction}

The digitalization of the economy in the past decades has made data availability grow and become more important. Within the maintenance field, new technologies are raising and the possibilities opened with the Internet of Things are countless. At the same time, customers are more demanding on maintenance performance and added value, wanting to reduce their exploitation costs while having no unplanned stops. Consequently, in recent years, advanced maintenance strategies, such as predictive maintenance (PdM) and prescriptive maintenance (PsM), gathered a lot of attention from researchers.

Although no formal and widely accepted definition of PsM can yet be found in the literature, it is understood as a maintenance integrated framework where the best action to take is quantitatively chosen, based on criteria that take into account all the functionalities of the system, Iung (2019). PsM differs from PdM because it considers a comprehensive set of actions for prescription, going beyond the maintenance actions classically considered in maintenance policies (e.g. repair, inspections and replacements), such as changing aspects of system usage to reduce the full exploitation cost.

One of the main difficulties of implementing PsM solutions is that prognostic models are usually built to estimate remaining useful life (RUL) Tang et al. (2014), but they do not take into account the decision making process that follows in real-life applications. Similarly, researchers who focus on decision making for maintenance consider prognostics as a given input, Lesobre et al. (2014), which does not always properly take into account the specificity of the prognostic information, as e.g. the impact of different decisions on the state of health of the system, etc.

Such a situation where the prognostics step and the health management process are not tightly interlinked may be limiting the performance of the

Proceedings of the 31th European Safety and Reliability Conference.

Edited by Bruno Castanier, Marko Cepin, David Bigaud and Christophe Berenguer 
whole prognostics and health management (PHM) loop, Atamuradov et al. (2017), and does not allow to make decisions to fully exploit the possible different usage modes for the system, to choose what tasks to prioritize and to reduce the overall exploitation cost.

PsM actions go beyond maintenance, inspection and replacement dates, and consider all functionalities and cost sources of a system. Going into this direction, Wang and Zhu (2021) have worked on deciding maintenance dates as well as deriving rules for inventory control, using degradation information to jointly manage replacement schedules and spare parts. Another example of expanding the usual maintenance decisions can be found in Obando et al. (2018) where the authors have developed methods to control the torque of a friction drive system in order to expand its RUL.

For industrial vehicles applications, a method for scheduling jointly both missions and maintenance operations for a fleet of vehicles was proposed by Robert et al. (2019); however, the proposed approach has not modelled important operational aspects of the problem and has not addressed the fact that, in practical problems, maintenance and systems exploitation decisions happen in two very different time scales. Those aspects will be addressed in this paper, and a full closed loop decision making algorithm for a particular vehicle component will be presented, scheduling maintenance and managing the vehicles of a fleet, and hence considering both the prognostic and the decision making problems.

Accordingly, the rest of the paper is organized as follows. Section 2 addresses the problem formulation, presenting its main aspects. Section 3 details the modeling of the system degradation and the different actions that can be applied to the fleet. Section 4 presents the full decision-making algorithm. In Section 5, the proposed approach is illustrated through simulations. Finally, Section 6 concludes the paper.

\section{Problem Statement}

The goal of the present work is to propose the optimal exploitation strategy for a fleet of vehicles, in order to provide fleet owners with the necessary tools for deciding which vehicles to send to a given mission, as well as when to perform maintenance operations.

Missions are defined as the deliveries that a vehicle has to make from one point to another. The points in space to be visited are associated with a deadline. All the distances from point to point are considered to be known, as well as the duration of the displacements from one point to another. Distances are stored in a matrix $D$ and durations in a matrix $T$.

Most vehicle owners have maintenance contracts that include periodic visits to workshops to perform routine maintenance operations such as oil changes and revisions. To avoid extra visits to the workshop - which would possibly lead to downtime and add costs - it is beneficial to perform necessary components replacements during those regular visits. The maintenance date decision comes down to choosing which of those already planned visits to use for components replacements.

There is a trade-off behind this decision-making process. If a component is not changed when it should have been, the owner takes the risk to have a breakdown which will create huge costs. On the other hand, if he changes it too early he wastes a still usable component. This trade-off will be addressed and quantified in the proposed model and decision-making scheme in future sections.

Both maintenance operations and mission planning are conversely connected. Sending vehicles that are more degraded to do less severe missions - those which are less likely to cause big increases in the degradation - can postpone maintenance operations. On the other hand, knowing that a given component will be changed for sure in the next visit to the workshop should also change the way missions are planned. If a vehicle owner is guaranteed to have a brand new component in a few days, he should use the current component as much as possible in this remaining period, avoiding wastes and sparing other vehicles.

The PsM solution goal is to provide the best mission schedule for a fleet of vehicles and decide maintenance dates, considering the effect one has on the other. In this work, we propose a PsM solution focused on a single critical component of a vehicle, i.e. the brake-pad.

We consider the following structured policy for a fleet of $M$ vehicles. At each decision time $\tau_{k}$, two decisions have to be made:

- Maintenance Date Decision : assuming that the two next scheduled workshop visits of vehicle $i$ will happen at $t_{w k p 1_{i}}$ and $t_{w k p 2_{i}}$, it is necessary to decide whether or not to replace the brake-pad at $t_{w k p 1_{i}}$, based on the current brake-pad thickness level, $Y_{k_{i}}$.

- Missions scheduling : missions are then dispatched among trucks so as to minimize an overall cost function

The model associated to this policy and its solution are developed in the next sections.

\section{Model Development}

\subsection{Working Assumptions}

The main working hypotheses are:

- A decision epoch $\tau_{k}$ arises when a new set of missions has to be accomplished. 


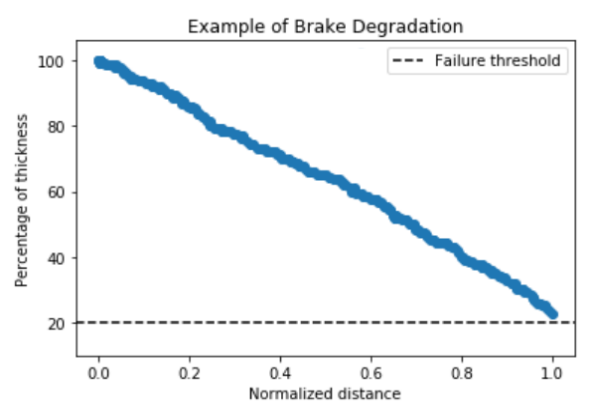

Fig. 1. Example of a brake pad degradation path

For example, every day a fleet owner has to make deliveries and therefore, decisions will happen at the beginning of each day, before sending vehicles from the headquarter.

- $T, D$ are known and displacements durations are considered to be deterministic. A version of this problem where distances and durations are considered as stochastic can be tackled in future works to have a more realistic solution.

- $t_{w k p 1_{i}}$ and $t_{w k p 2_{i}}$ are known for every vehicle $i$ at $\tau_{k}$ as well as $Y_{k}$. Both $t_{w k p 1_{i}}$ and $t_{w k p 2_{i}}$ are determined by a maintenance contract and therefore, no decision making process can affect those quantities.

- Only immobilizations caused by problems with the brake-pad are considered.

\subsection{Component Deterioration Model}

The brake system is critical in any vehicle. One of its key elements is the brake-pad, a component of disk brakes used in several applications. Due to its simplicity and importance,the brake-pad has been chosen as a use case for this work.

The most common failure mode of the brakepad is due to wear and tear with usage. A brakepad is considered defective whenever its thickness falls under a given threshold. In this paper, this event is considered as a failure and will require the vehicle to be stopped.

Fig. 1 shows an example of how thickness evolved for a brake-pad.

For a given mode of operation, the brake-pad thickness evolution exhibits a mean trend close to be directly proportional to the traveled distance, but also a temporal and item-to-item variability. One of the most common classes of stochastic process used to model this type of degradation phenomena is the Wiener process with a linear drift Tang et al. (2014). In this paper, the chosen version of such a model is:

$$
Y(x)=Y_{0}+\lambda x+\sigma_{B} B(x)+\epsilon
$$

where $Y$ is the brake-pad thickness, $x$ is the traveled distance, $Y_{0}$ is the initial thickness, $\lambda$ is the negative drift, considered to be constant and estimated through data, $B(x)$ is the standard Brownian motion, $\sigma_{B}$ is its variance and $\epsilon$ is a measurement noise considered to be Gaussian. Those parameters can be estimated directly from data as explained in Tang et al. (2014).

This model correlates degradation to traveled distance and therefore can be used to connect degradation and mission planning, as well as to base the maintenance date decision, as it will be seen in the following sections.

\subsection{Maintenance Date Decision}

We assume that, at a decision epoch $\tau_{k}$, the two next scheduled dates of workshop visit $t_{w k p 1_{i}}$ and $t_{w k p 2_{i}}$ are known for each vehicle $i$. The maintenance decision at hand is then to choose whether the next workshop visit date $t_{w k p 1_{i}}$ has to be retained for a brake-pad replacement or whether this operation can be postponed at least until $t_{w k p 2_{i}}$. If a replacement happens too early at $t_{w k p}$, a cost related to the wasted thickness is incurred:

$$
\frac{C_{b k}}{Y_{0}}\left(Y\left(t_{w k p}\right)-Y_{c r i t}\right)
$$

where $Y\left(t_{w k p}\right)$ is the measured thickness at the moment of the replacement and $Y_{c r i t}$ is the critical failure threshold of a single brake-pad.

It is important to highlight that, differently from delay, distance and degradation costs, Eqn. 2 is not computed on the fleet level, but evaluated independently for every vehicle.

Deciding not to change the brakes at $t_{w k p 1}$ and to wait until $t_{w k p 2}$ corresponds to a risk of a vehicle immobilization between the present date and $t_{w k p 2}$ whose cost can be estimated by:

$$
C_{\text {fail }} \mathbb{P}\left[Y\left(t_{w k p 2}\right)<Y_{c r i t} \mid Y\left(t_{w k p 1}\right)\right]
$$

where $\mathbb{P}\left[Y\left(t_{w k p 2}\right)<Y_{c r i t} \mid Y\left(t_{w k p 1}\right)\right]$ is the probability of failing before $t_{w k p 2}$, given that $Y\left(t_{w k p 1}\right)$ is known and $C_{\text {fail }}$ is the total cost of an immobilization, which accounts for corrective maintenance costs and the impact of the immobilization on the deliveries, including extra delays, the cost of changing other vehicles plans throughout the work session, etc.

A brake-pad will be replaced at $t_{w k p 1}$ if:

$$
\begin{array}{r}
\frac{C_{b k}}{Y_{0}}\left(Y\left(t_{w k p 1}\right)-Y_{c r i t}\right) \\
<C_{f a i l} \mathbb{P}\left[Y\left(t_{w k p 2}\right)<Y_{c r i t} \mid Y\left(t_{w k p 1}\right)\right]
\end{array}
$$

\subsection{Costs for Missions Assignment}

In the following section, the costs used in the optimization of mission schedules are presented. 
It is important to keep in mind that some of those costs are not monetarily paid at every $\tau_{k}$, but guide the optimization process to reduce the long term exploitation cost.

\subsubsection{Degradation Cost}

Whenever a fleet of vehicles follows a mission schedule, the expected average thickness of the fleet decreases. The total thickness variation over the fleet can be expressed as:

$$
\sum_{i=1}^{N} Y_{k_{i}}-\sum_{i=1}^{N} E\left[Y_{k+1_{i}}\right]=\sum_{i=1}^{N} \lambda_{i} X_{i}
$$

where $N$ represents the vehicles that will not undergo brake-pad replacements in the next workshop visit (vehicles with a maintenance date decided must be used as much as possible before replacement). $Y_{k_{i}}$ is the thickness of the brake-pad of vehicle $i$ before performing the scheduled missions, $Y_{k+1_{i}}$ is the thickness of the brake-pad of vehicle $i$ after performing the scheduled missions, since this quantity is stochastic, the expectation operator $E$ is used. $X_{i}$ is the distance vehicle $i$ will travel while following this mission schedule.

Therefore, the fleet expected degradation cost of a mission schedule can be written as:

$$
C_{d e g}=\frac{C_{b k}}{Y_{0}} \sum_{i=1}^{N} \lambda_{i} X_{i}
$$

where $C_{b k}$ is the cost of a brake-pad.

\subsubsection{Operational Costs}

Traditionally, scheduling missions is mainly impacted by fuel consumption and deadlines. To be able to quantitatively decide between two different schedules we have to model those cost sources.

As the focus of this article lies in the optimization of planning and maintenance, fuel consumption costs are considered to be directly proportional to the traveled distance. In the future, this PsM algorithm can be easily adapted to account for offline consumption estimations similar to Basso et al. (2019). For now, the cost related to distances $C_{d i s t}$ is given by:

$$
C_{d i s t}=C_{K m} \sum_{i=1}^{M} X_{i}
$$

where $C_{K m}$ is a fuel price based constant, $M$ represents the whole fleet, and $X_{i}$ is the distance that will be traveled by vehicle $i$.

While planning missions, it is important to consider the deadlines. In real life, penalties for late deliveries can be applied in several different ways. In this paper, penalties are considered to be proportional to the delay. Therefore, the delay cost is:

$$
C_{\text {delay }}=C_{d} \sum_{i=1}^{P} \max \left[t_{i}-l_{i}, 0\right]
$$

where $C_{d}$ is a constant that is related to the cost of penalties, $P$ is the total number of points to be visited, $t_{i}$ is the arrival time at point $i$ and $l_{i}$ is the deadline of point $i$. Notice that in this formulation, the duration of the displacement between two points is considered to be deterministic, so, once mission plan is decided, the arrival instant $t$ is known. This assumption means that uncertainties on the delivery date caused by traffic or unplanned breakdowns are not considered, which is justified since this paper considers only immobilizations due to brake-pad wear, which should not occur while following the PsM schedules.

$C_{\text {dist }}$ and $C_{\text {delay }}$ are completely defined by distance matrix $D$, duration matrix $T$, the deadlines of each delivery point and a mission plan.

\subsubsection{Waste Cost}

As explained in Section 2, once a maintenance date is decided for a given vehicle, it must be used as much as possible before the replacement, avoiding waste and sparing other vehicles. To do so, a dynamic cost is used. Its construction starts by defining $t^{*}$.

$$
t_{i}^{*}=t-t_{\text {maint }_{i}}
$$

where $t_{\text {maint }_{i}}$ is the maintenance date of vehicle $i$, and $t$ is the present time. Then the cost of not using vehicles that will undergo brake replacements, $C_{w}$, is:

$$
C_{w}=\frac{C_{b k}}{Y_{0}} \sum_{i=1}^{N^{*}}\left(E\left[Y_{i}\right]-Y_{c r i t}\right) A\left(t_{i}^{*}\right)
$$

where $N^{*}$ are the vehicles that will undergo a brake-pad change at $t_{\text {maint }}, E\left[Y_{i}\right]$ is the expected thickness of vehicle $i$ after completing the scheduled missions, $Y_{\text {crit }}$ is the critical failure threshold of a brake-pad and $A\left(t_{i}^{*}\right)$ is an activation function. Similarly to the degradation, delay and distance cost, $C_{w}$ is computed at the fleet level.

$A\left(t_{i}^{*}\right)$ could be any monotonic increasing function, so that it grows with $t^{*}$, meaning that the cost of not using the vehicle becomes more important the closer the replacement date is. The chosen activation function is:

$$
A\left(t^{*}\right)=S\left(\alpha t^{*}+\beta\right)
$$




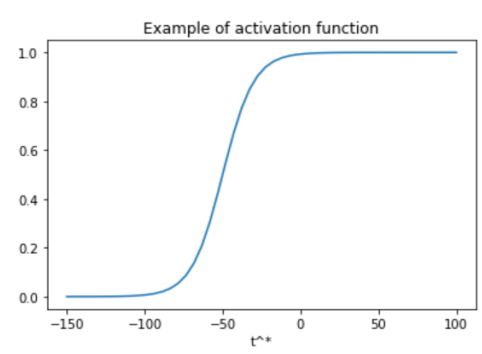

Fig. 2. Example of an activation function

where $\alpha$ and $\beta$ are positive constants and $S$ is the sigmoid function. $A\left(t^{*}\right)$ is represented in Fig 2 .

Notice that $N^{*}$ accounts for the vehicles that will undergo replacements and should be more used, while $N$ considers those vehicles for which a replacement date was not yet defined, and therefore, whose thickness should be spared and maximized, and finally $M$ is the ensemble of all vehicles in the fleet.

$C_{w}$ is a dynamic cost, depending on the maintenance date. Minimizing it will make vehicles that will have their brake-pad changed, more used, avoiding waste and sparing other vehicles.

\section{Closed Loop Decision Making}

Based on the model developed in the previous section, a closed loop PsM decision making procedure for the joint maintenance and missions assignment can now be implemented. The PsM solution is represented in Fig 3

The solution is composed of two main parts, a scheduler and a maintenance date decision maker. The scheduler will receive the list of missions to be accomplished, alongside with the related information (matrices $D, T$ and respective deadlines), and the last available degradation measurement. It will then proceed to find the best schedule possible, in order to reduce the long-term overall exploitation cost.

This mission schedule will be followed by the fleet and new degradation measures will be collected. These measurements will be used as an input by the maintenance date decision maker which will decide if a vehicle will replace its brake-pads in the next workshop visit. Replacement dates are then updated and used to change the scheduler cost function.

In the following sections, the scheduler and the maintenance decision maker are explained in detail.

\subsection{Scheduler}

The scheduler is responsible for finding the optimal mission schedule $\pi$. The optimization criterion that accounts for the overall exploitation

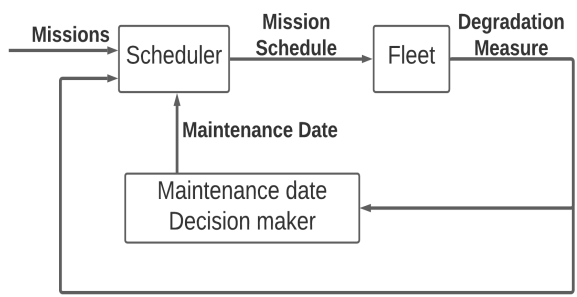

Fig. 3. PsM structure

cost $C(\pi)$ integrates all the costs evaluated in the previous section:

$$
\begin{array}{r}
C(\pi)=C_{\text {delay }}+C_{\text {dist }}+ \\
C_{w}+C_{\text {deg }}
\end{array}
$$

In a fleet with identical vehicles, minimizing $C_{d e g}$ implies minimizing the traveled distance, and therefore, in terms of Eq. 12, any schedule that reduces the total distance and respect deadlines will be equivalent. On the other hand, it is beneficial not only to reduce the total degradation cost, but also to use less degraded vehicles to perform shorter missions, postponing brake-pad replacements. To address this fact, the optimization problem is:

$$
\min _{\pi} C(\pi)-\gamma Y_{\min }
$$

where $\gamma$ is a positive constant, determined empirically, through simulations and $Y_{\min }$ is the minimal expected thickness in the fleet, after following the mission plan $\pi$. The term $-\gamma Y_{\min }$ will privilege schedules that not only reduce the overall exploitation cost but also postpone maintenance operations, using less degraded vehicles to perform more demanding missions.

Since this problem of scheduling missions is a variation of the travel salesman problem, it is NP-hard. The optimizer used was based on genetic programming, a heuristic method that, even though fast, is not guaranteed to find the optimal solution every time.

\subsection{Maintenance Date Decision Algorithm}

To make the decision of choosing the next workshop visit for brake-pad replacement, it is needed to evaluate Eq. 4 and thus to estimate $\mathbb{P}\left[Y\left(t_{w k p 2}\right)<Y_{c r i t}\right]$ and $Y\left(t_{w k p 1}\right)$. To this aim, it is necessary to make the hypothesis that $\mathbb{P}[X / T]$ - distribution of the distance traveled given a specific time window - is known, since $Y$ correlates with the distance traveled by a vehicle and not time.

Once $\mathbb{P}[X / T]$ is known, Monte-Carlo methods can be used to decide the maintenance date. 
Table 1. Fleet initial conditions

\begin{tabular}{llll}
\hline & $\begin{array}{l}Y_{0} \\
(\%)\end{array}$ & $\begin{array}{l}\lambda \\
\left(\mathrm{km}^{-1}\right)\end{array}$ & $\begin{array}{l}\sigma_{B} \\
\left(\mathrm{~km}^{-1}\right)\end{array}$ \\
\hline Vehicle 1 & 100 & $-1 \mathrm{e}-3$ & $1 \mathrm{e}-1$ \\
Vehicle 2 & 100 & $-8 \mathrm{e}-4$ & $1 \mathrm{e}-1$ \\
Vehicle 3 & 100 & $-8 \mathrm{e}-4$ & $1 \mathrm{e}-1$ \\
Vehicle 4 & 100 & $-8 \mathrm{e}-4$ & $1 \mathrm{e}-1$ \\
\hline
\end{tabular}

Whenever a degradation measure is available and therefore the current value of $Y$ is known, the algorithm estimates the average $Y\left(t_{w k p 1}\right)$ by making random samplings of $\mathbb{P}[X / T]$ to infer the average distance traveled at $t_{w k p 1}$. Similarly, $\mathbb{P}\left[Y\left(t_{w k p 2}\right)<Y_{c r i t}\right]$ is estimated using random samples of $\mathbb{P}[X / T]$, in that case, the number of trajectories in which an immobilization occurred before $t_{w k p 2}$ is compared to the number of trajectories in which it did not, so that this probability can be empirically estimated.

In real life applications, $\mathbb{P}[X / T]$ can be estimated based on the vehicle's usage history and the client's business model.

\section{Numerical Experiments and Results}

To test the PsM solution, a comparison between the proposed algorithm and other exploitation strategies is made through simulations, to see how they can affect the long term cost.

\subsection{Missions and Fleet Parameters}

The simulations emulate the exploitation of a fleet of vehicles that have to perform the same missions every week. Those fleets are identical and have the parameters presented on Table 1 .

Matrix $D$ and $T$ are chosen so that every point in space is equidistant (equals to $100 \mathrm{~km}$ ) and every possible displacement has the same duration (equals to 1 hour). This choice is done in order to have a configuration where different schedules would lead to optimal distance and delay costs. In such configuration, the scheduler can have more impact on the costs related to degradation and maintenance since more schedules are acceptable in terms of delay and distance costs. The set of missions chosen is presented in Table 2 . Point 0 has no deadline attached, representing a headquarters, from where vehicles will have to start their missions and to where they will go back after finishing them. Points 5 to 11 should be visited until 12 hours after the vehicles depart from the headquarters. Points 1 to 4 have tighter deadlines in order to force all the vehicles to be used.

The constants used in the optimization cost function are given in Table 3
Table 2. Point to be visited

\begin{tabular}{ll}
\hline Point & Deadline (h) \\
\hline Point 0 & - \\
Points $1,2,3 \& 4$ & 1 \\
Points $5,6,7,8,9,10 \& 11$ & 12 \\
\hline
\end{tabular}

Table 3. Normalized Constant Values For Simulations

\begin{tabular}{ll}
\hline Constant & Value \\
\hline$C_{b k}$ & 1 \\
$C_{K m}$ & 0.0015 \\
$C_{d}$ & 0.4 \\
$C_{\text {fail }}$ & 14.2 \\
$Y_{\text {crit }}$ & 0 \\
\hline
\end{tabular}

In the simulation, whenever a truck performs a schedule of missions, the thickness of the brakepad evolves according to the model (1).

\subsection{Fleet strategies}

Three different strategies are compared through simulations, all of them start in the same initial conditions, presented at table (1) and have to perform the missions of Table 2 every week, scheduling mission and brake-pad replacements in different ways:

Strategy 1 consists in using the PsM algorithm in close-loop as represented in Fig 3 . To decide replacement dates, $\mathbb{P}[X / T]$ must be estimated. This distribution is obtained through a first simulation, performed with different degradation levels for a fleet with the initial parameters of Table 1

Strategy 2 emulates a strategy used by some real clients. Every week, the fleet follows the same schedule. This fixed schedule does not take the degradation model into consideration, only accounting for delay and distance costs and minimizing the total duration of missions. This schedule then minimizes the total distance, the delays and finishes all deliveries as soon as possible, as real fleet managers do. Clients with certain types of maintenance contract receive an alert to schedule brake-pad replacements whenever thickness falls under a warning threshold $Y_{w}$. To emulate a client maintenance strategy, replacements will occur whenever a regular visit to the workshop happens and thickness is already below $Y_{w}$.

Strategy 3 consists in using an open-loop version of the PsM algorithm. A schedule of missions is built using the PsM scheduler once, therefore taking into account the degradation parameters of 
Table 4. Fixed Schedule - Strategy 2

\begin{tabular}{ll}
\hline & Points Visited \\
\hline Vehicle 1 & $0,3,10,6,0$ \\
Vehicle 2 & $0,1,9,11,0$ \\
Vehicle 3 & $0,2,5,7,0$ \\
Vehicle 4 & $0,4,8,0$ \\
\hline
\end{tabular}

Table 5. Fixed Schedule - Strategy 3

\begin{tabular}{ll}
\hline & Points Visited \\
\hline Vehicle 1 & $0,1,0$ \\
Vehicle 2 & $0,2,5,7,0$ \\
Vehicle 3 & $0,3,6,10,11,0$ \\
Vehicle 4 & $0,4,9,8,0$ \\
\hline
\end{tabular}

each truck. This schedule will be followed every week. Maintenance will happen in the same way as for strategy 2, whenever a regular visit to the workshop happens and thickness is already below $Y_{w}$.

\subsection{Simulation Analysis}

\subsubsection{Schedules}

Table 4 shows fixed schedule followed by Strategy 2. It completes the missions as fast as possible, and since all points are equidistant and it starts with the point that presents the earlier deadlines, both distance and delay costs are optimized. On the other hand, since this planning does not take into account the degradation cost, it uses Vehicle 1 - which has the highest absolute deterioration rate $|\lambda|$ - to perform more missions than necessary. The normalized degradation cost associated with this schedule is $C_{d e g}=0.005$.

With the fixed schedule followed by Strategy 3 presented in Table 5, it can be seen that, similarly to Strategy 2, this schedule minimizes distance traveled and does not cause any delay. On the other hand, since this schedule was found by the PsM scheduler, it takes degradation into account and therefore, avoids using Vehicle 1 as much as possible.

Strategy 1 does not follow a fixed schedule. Fig 4 shows the histogram of traveled distances for each vehicle of the fleet. It can be seen that Vehicle 1 was not used for more than one mission in most weeks, reducing the degradation cost to $C_{d e g}=0.003$. The schedules of missions in which it is used more than once can be explained by the fact that the genetic algorithm does not converges to the optimal schedule every time and

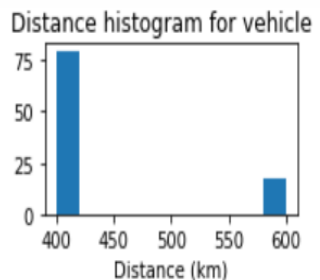

Distance histogram for vehicle 2
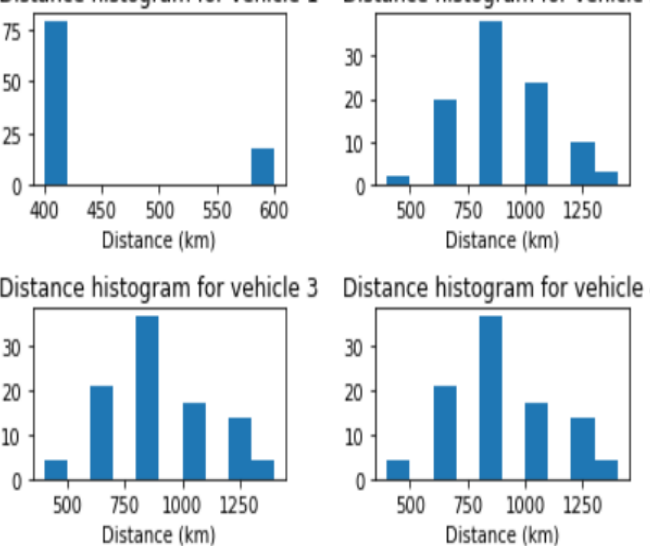

Distance histogram for vehicle 4

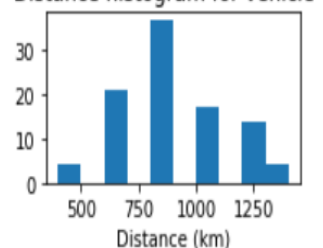

Fig. 4. Histogram of traveled distances - Strategy 1

in a few occasions, due to the randomness of the algorithm, a sub-optimal schedule was used.

\subsubsection{Long Term Cost}

To compare the different strategies, it is necessary to compute the long term exploitation cost, which is composed of delay, distances and maintenance costs. Delay and distances costs are obtained directly from $\mathrm{Eq} 7$ and $\mathrm{Eq} .8$

$C_{d e g}$ is not a cost paid daily by fleet managers, it is only a measure of how well exploited the fleet was in terms of health management.

The real cost paid by fleet managers is the replacement and immobilization costs.

In the simulations, whenever a replacement of brake-pad happens, maintenance cost is increased by $C_{b k}$ and whenever an immobilization happens, maintenance cost is increased by $C_{f a i l}$. Notice that the only possible cause for immobilization considered is related to the brake-pad wear, not accounting for failures cause by other components. $C_{\text {fail }}$ includes all the expenses related to the immobilization, including extra delays, so that, adding it to the simulation cost is its only consequence.

Operational costs are identical for the three fleets. No delay cost is payed and distance cost is equal every week to $C_{d i s t}=0.19$, which means that the scheduler is able to reduce the traveled distance and the delay for the fleet in every mission schedule.

Figures 5 and 6 show the maintenance cost and the waste cost (as calculated in Eq. 2).

We can notice that no immobilization occurred with any of the strategies. On the other hand, with Strategy 2, more replacements were necessary than for the other strategies. This is due to the fact that its fixed schedule has a higher $C_{d e g}$ and shows that considering the knowledge on the 


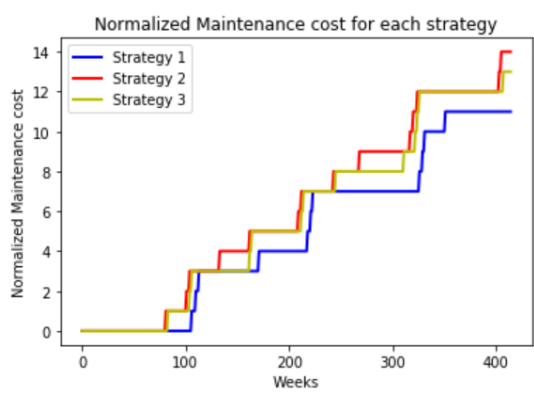

Fig. 5. Maintenance Cost Comparison

deterioration rate $\lambda$ and on the deterioration level while scheduling missions can drastically improve the long term exploitation cost.

Strategy 2 and Strategy 1 use schedules derived from the PsM algorithm. Even following sub-optimal schedules sometimes, Strategy 1 performed better, postponing maintenance operations and avoiding waste. This is due to the use of dynamic scheduling that accounts for maintenance dates and often uses more degraded vehicles to perform the least demanding missions.

\section{Conclusion and future work}

In this paper, a PsM solution was proposed for a fleet of vehicles. The actions to be analyzed were related to the plan of mission and the maintenance date, considering the impacts one has on the other. The component chosen for this task was the brake-pad, modeled with a wiener process. While scheduling missions, different operational costs that can be inferred from basic mission characteristics and from the degradation model, were modelled. For the maintenance date part, a MonteCarlo method was used, based on the hypothesis that $\mathbb{P}[X \mid T]$ is known. To improve it, methods for online estimation of $\mathbb{P}[X \mid T]$ can be added in the future. Also, in future works a stochastic version of matrix $\mathrm{D}$ and $\mathrm{T}$ should be considered. Finally, through simulation, the PsM solution was compared to two different exploitation strategies, showing it outperforms the strategy 2 and that its usage on close loop can outperform its usage in open loop (Strategy 3). Because of the probabilistic nature of the degradation phenomena, more tests are needed to quantify exactly how much improvement is achieve through the PsM solution.

In future works, more complex operational constraints can be studied and component models that allow for more complex actions, such as parameters changes, replacements for used components, etc, may be used.

\section{References}

Atamuradov, V., K. Medjaher, P. Dersin, B. Lamoureux, and N. Zerhouni (2017). Prognos-

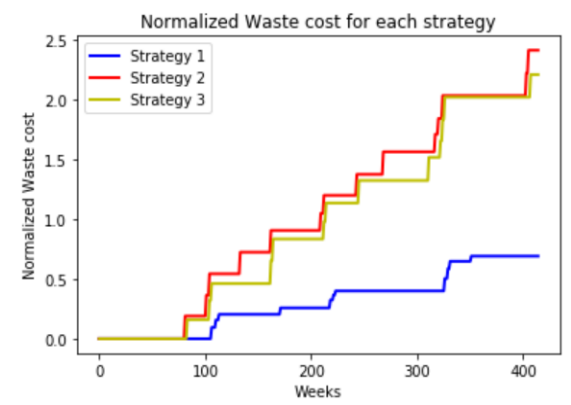

Fig. 6. Waste Cost Comparison

tics and health management for maintenance practitioners-review, implementation and tools evaluation. International Journal of Prognostics and Health Management, 1-31.

Basso, R., B. Kulcsár, B. Egardt, P. Lindroth, and I. Sanchez-Diaz (2019). Energy consumption estimation integrated into the electric vehicle routing problem. Transportation Research Part D: Transport and Environment 69, 141-167.

Iung, B. (2019). De la maintenance prédictive à la maintenance prescriptive: Une évolution nécessaire pour l'industrie du futur. In Conference on Complexity Analysis of Industrial Systems and Advanced Modeling, CAISAM 2019.

Lesobre, R., K. Bouvard, C. Bérenguer, A. Barros, and V. Cocquempot (2014). A usage-informed preventive maintenance policy to optimize the maintenance free operating period for multicomponent systems. In Probabilistic Safety Assessment and Management (PSAM 12).

Obando, D. R., J. J. M. Molina, and C. Bérenguer (2018). An architecture for controlling the remaining useful lifetime of a friction drive system. In 10th IFAC Symposium on Fault Detection, Supervision and Safety for Technical Processes, pp. 861-866.

Robert, E., K. Bouvard, R. Lesobre, and C. Bérenguer (2019). Joint assignment of missions and maintenance operations for a fleet of deteriorating vehicles. In Proc. of 11th International Conference on Mathematical Methods in Reliability - MMR2019, City University HongKong, Jun 2019, Hong-Kong, China.

Tang, S., X. Yu, C.and Wang, and X. Guo, X.and Si (2014). Remaining useful life prediction of lithium-ion batteries based on the Wiener process with measurement error. Energies, 520-547.

Wang, J. and X. Zhu (2021). Joint optimization of condition-based maintenance and inventory control for a k-out-of-n:f system of multi-state degrading components. European Journal of Operational Research 290, 514-529. 